\title{
HOMOGENEUS MIXED PROBLEM FOR THE DAMPED CARRIER EQUATION
}

\author{
L.P. San Gil Jutuca, \\ R.F. Apolaya ${ }^{2}$, \\ M. Milla Miranda ${ }^{3}$
}

Key Words: Carrier equation, Galerkin method, Homogeneous mixed problem.

\section{ABSTRACT}

This paper is concerned with the existence of global solutions of an initial and homogeneous boundary problem for the damped Carrier equation

$$
\frac{\partial^{2} u}{\partial t^{2}}-M\left(\int_{\Omega}|u|^{2} d \Omega\right) \Delta u+\left|\frac{\partial u}{\partial t}\right|^{\rho} \frac{\partial u}{\partial t}=0
$$

where $M$ is a positive real function and $\rho>1$.

\section{Introduction}

Let $\Omega$ be a bounded open set of $R^{n}$ with boundary $\Gamma$ of class $C^{2}$. We consider a partition $\left\{\Gamma_{0}, \Gamma_{1}\right\}$ of $\Gamma$ such that $\Gamma_{1}$ is open in $\Gamma$, mes $\left(\Gamma_{1}\right)>0$, mes $\left(\Gamma_{0}\right)>0$ and $\bar{\Gamma}_{0} \cap \bar{\Gamma}_{1} \neq \phi$. In this paper, the authors investigate, by using Galerkin's method, the existence and uniqueness of global solutions for the following mixed problem:

$$
\begin{aligned}
& u^{\prime \prime}-M\left(\int_{\Omega}|u|^{2} d \Omega\right) \Delta u+\left|u^{\prime}\right|^{\rho} u^{\prime}=0 \text { in } \Omega \times[0, \infty), \\
& u=0 \text { on } \Gamma_{0} \times[0, \infty), \\
& \frac{\partial u}{\partial v}+\delta u^{\prime}=0 \text { on } \Gamma_{1} \times[0, \infty), \\
& u(x, 0)=u^{0}(x), u^{\prime}(x, 0)=u^{\prime}(x) \text { in } \Omega
\end{aligned}
$$


Here $M(\lambda)$ is a positive real function of class $C^{1}$ on $[0, \infty)$; the vector $v$ denotes an outward unit normal to the boundary $\Gamma$ and $\delta$ is a function in $W^{1, \infty}(\Gamma)$ such that $\delta(x) \geq 0$. By $u^{\prime}, u^{\prime \prime}$ we denote the time derivatives of $u$.

Global solutions for equation (1.1) with null Dirichlet boundary were obtained by C. L. Frota, A. T. Cousin and N. Larkin [3] and for the Kirchhoff-Carrier equation without damping by A. T. Cousin, C. L. Frota, N. Larkin and L. A. Medeiros [2].

\section{Preliminaries}

In order to formulate our results we consider the Hilbert space

$$
V=\left\{v \in H^{1}(\Omega) ; v=0 \text { on } \Gamma_{0}\right.
$$

with inner product and norm given by:

$$
((u, v))=\sum_{i=1}^{n} \int_{\Omega} \frac{\partial u}{\partial x_{i}}(x) \frac{\partial v}{\partial x_{i}}(x) d x \text { and }\|u\|=\left(\sum_{i=1}^{n} \int_{\Omega}\left(\frac{\partial u}{\partial x_{i}}(x)\right)^{2} d x\right)^{1 / 2}
$$

The inner product and norm of $L^{2}(\Omega)$ are represented by (.,.) and $|$.$| , respectively.$

Let $\mathrm{W}$ be the space of functions $u: \Omega \rightarrow R$ such that $u \in V, \Delta u \in L^{2}(\Omega)$ and there is $g_{u} \in H^{1 / 2}(\Gamma)$ which satisfies $g_{u} \equiv 0$ on $\Gamma_{0}$ and

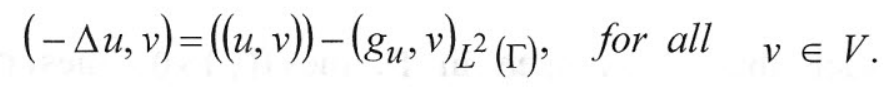

We remark that $g_{u}$ verifying (2.1) is unique. The space $\mathrm{W}$ is equipped with the norm

$$
\|u\|_{w}=\left(|\Delta u|^{2}+\left\|g_{u}\right\|_{H^{1 / 2}(\Gamma)}^{2}\right)^{1 / 2}
$$

Then W is a separable Hilbert space and W is compactly embedding into V.

Proposition 2.1 The space $W$ is dense in $V$.

The proof of this Proposition, based on the density of $D(-\Delta)$ in $\mathrm{V}$, is reasonably straightforward and follows arguments close to the used in [4]. 
Remark 2.1 If $u \in W$ then $\frac{\partial u}{\partial v} \in H^{-1 / 2}(\Gamma)$ and it holds that

$$
\left\langle\frac{\partial u}{\partial v}, v\right\rangle_{H^{-1 / 2}(\Gamma), H^{1 / 2}(\Gamma)}=\left(g_{u}, v\right)_{L^{2}(\Gamma)} \quad \text { for all } \quad v \in V .
$$

This implies by taking $v \in D\left(\Gamma_{1}\right)$ that

$$
\frac{\partial u}{\partial v}=g_{u} \quad \text { in } \quad H^{1 / 2}\left(\Gamma_{1}\right)
$$

\section{Main Result}

In order to obtain the existence of global solutions of Problem (1.1) - (1.4), we assumed the following supplementary assumptions on $M$ :

$$
\begin{gathered}
M(\lambda) \geq m_{0}>0 \quad\left(m_{0} \text { is constant }\right) \\
\frac{\left|M^{\prime}(\lambda)\right|}{M(\lambda)} \lambda^{1 / 2} \leq K_{0}
\end{gathered}
$$

where $M^{\prime}(\lambda)$ denotes the derivative of $M$ with respect to $\lambda$ and $K_{0}$ is a constant.

Remark 3.1 If we consider smallness restrictions on the initial data $u^{0}$ and $u^{1}$, then above hypothesis (3.2) on the function $M$ becomes unnecessary .

We also impose on the real number $\rho$ the conditions:

$$
\mid \begin{aligned}
& \rho>1 \quad \text { if } \quad n=2 \\
& 1<\rho \leq \frac{n+2}{n-2} \quad \text { if } \quad n \geq 3
\end{aligned}
$$

Theorem 3.1 Assume that the conditions (3.1) - (3.3) are satisfied and that $u^{0} \in V, u^{1} \in V$ verify $\Delta u^{0} \in L^{2}(\Omega)$,

$$
\frac{\partial u^{0}}{\partial v}+\delta u^{1}=0 \quad \text { in } \quad H^{1 / 2}\left(\Gamma_{1}\right)
$$


Then there exists a unique function $u: \Omega \times] 0, \infty[\rightarrow R$ in the class

$$
\begin{gathered}
u \in L_{l o c}^{\infty}(0, \infty ; W), \quad u^{\prime} \in L_{l o c}^{\infty}(0, \infty ; V), \quad u^{\prime \prime} \in L_{l o c}^{\infty}\left(0, \infty ; L^{2}(\Omega)\right), \\
\delta^{1 / 2} u^{\prime \prime} \in L_{l o c}^{2}\left(0, \infty ; L^{2}\left(\Gamma_{1}\right)\right)
\end{gathered}
$$

satisfying the equation

$$
u^{\prime \prime}-M\left(|u(.)|^{2}\right) \Delta u+\left|u^{\prime}\right|^{\rho} u^{\prime}=0 \quad \text { in } \quad L_{l o c}^{\infty}\left(0, \infty ; L^{2}(\Omega)\right)
$$

and the initial conditions

$$
u(0)=u^{0}, \quad u^{\prime}(0)=u^{1}
$$

Furthermore u verifies

$$
\begin{aligned}
& \frac{\partial u}{\partial v}+\delta u^{\prime}=0 \quad \text { in } \quad L_{l o c}^{\infty}\left(0, \infty ; H^{1 / 2}\left(\Gamma_{1}\right)\right), \\
& \frac{\partial u^{\prime}}{\partial v}+\delta u^{\prime \prime}=0 \quad \text { in } \quad H_{l o c}^{-1}\left(0, \infty ; L^{2}\left(\Gamma_{1}\right)\right),
\end{aligned}
$$

Remark 3.2 If $u^{0}$ is in the conditions of Theorem 3.1 then $u^{0} \in W$ and

$$
\left(-\Delta u^{0}, v\right)=\left(\left(u^{0}, v\right)\right)+\left(\delta u^{1}, v\right)_{L^{2}(\Gamma)}, \text { for all } v \in V .
$$

The next result has a fundamental role in the proof of Theorem 3.1.

Lemma 3.1. Let us suppose that $u^{0} \in V, \Delta u^{0} \in L^{2}(\Omega)$ and $u^{1} \in V$ with

$$
\left(-\Delta u^{0}, v\right)=\left(\left(u^{0}, v\right)\right)+\left(\delta u^{1}, v\right)_{L^{2}(\Gamma)}, \text { for all } v \in V .
$$

Let $\varepsilon>0$. Then there exist $w$ and $z$ in $W$ such that

$$
\begin{gathered}
(-\Delta w, v)=((w, v))+(\delta z, v)_{L^{2}(\Gamma)}, \text { for all } v \in V \\
\left\|w-u^{0}\right\|_{w}<\varepsilon,\left\|z-u^{1}\right\|<\varepsilon .
\end{gathered}
$$

Proof. Fixe $\varepsilon>0$. By Proposition 2.1, there exists $z \in W$ such that $\left\|z-u^{1}\right\|<\varepsilon$. Let $w$ be the solution of the variational problem

$$
\mid \begin{aligned}
& w \in V \\
& ((w, v))=\left(-\Delta u^{0}, v\right)+(\delta z, v)_{L^{2}(г)} \text { for all } v \in V .
\end{aligned}
$$


Then $\Delta w=\Delta u^{0} ;$ moreover

$$
\begin{gathered}
\left\|w-u^{0}\right\|_{W}^{2}=\left|\Delta w-\Delta u^{0}\right|^{2}+\left\|-\delta z+\delta u^{1}\right\|_{H^{1 / 2}(\Gamma)}^{2} \\
\leq C\left\|z-u^{1}\right\|_{H^{1 / 2}(\Gamma)}^{2} \\
\leq C_{1}\left\|z-u^{1}\right\|^{2} \leq C_{1} \varepsilon^{2}
\end{gathered}
$$

where $C_{1}$ is a positive constant that depends only of $\delta$ and $\Omega$. Thus $w, z \in W$ and

$$
(-\Delta w, v)=((w, v))+(\delta z, v)_{L^{2}(\Gamma)} \text {, for all } v \in V \text {. }
$$

Proof of Theorem 3.1 From Lemma 3.1 there exist sequences $\left(u_{\ell}^{0}\right)$ and $\left(u^{1}\right)_{\ell}$ of vectors belonging to $W$ such that

$$
\begin{aligned}
& u_{\ell}^{0} \rightarrow u^{0} \text { strongly in } W \\
& u_{\ell}^{1} \rightarrow u^{1} \text { strongly in } V \\
& \left(-\Delta u_{\ell}^{0}, v\right)=\left(\left(u_{\ell}^{0}, v\right)\right)+\left(\delta u_{\ell}^{1}, v\right)_{L^{2}(\Gamma)} \text {, for all } v \in V
\end{aligned}
$$

From above sequences, for each $\ell \in N$, we construct a special basis of $W$ in the following way: first, we determine a orthonormal basis $w_{k}^{\ell}$ of the subspace of $W$ spanned by $u_{\ell}^{0}$ and $u_{\ell}^{1}$ ( $\ell$ fixed). Thus $k=1$ or $k=1,2$. Then by the orthonormalization process, we complete $\left(w_{k}^{\ell}\right)$ just to obtain a basis of $W$. This special basis of $W$ is represented by

$$
\left\{w_{1}^{\ell}, w_{2}^{\ell}, \ldots, w_{j}^{\ell}, \ldots\right\}
$$

In what follows $\ell$ is fixed, unless we mention the contrary. For $m \in N$ let us consider the subspace $W_{m}^{\ell}$ spanned by $\left\{w_{1}^{\ell}, w_{2}^{\ell}, \ldots, w_{m}^{\ell}\right\}$ and the approximate solutions $u_{l m}(t)$ of Problem (1.1) - (1.4), defined by

$$
u_{l m}(t)=\sum_{j=1}^{m} g_{l j m}(t) w_{j}^{\ell},
$$

where $g_{l j m}$ are the solutions of the approximate equation

$$
\begin{aligned}
& \left(u_{\ell m}^{\prime \prime}(t), w\right)+M\left(\left|u_{\ell m}(t)\right|^{2}\right)\left(\left(u_{\ell m}(t), w\right)\right)+ \\
& M\left(\left|u_{\ell m}(t)\right|^{2}\right) \int_{\Gamma 1} \delta u_{\ell m}^{\prime}(t) w d \Gamma+\left(\left|u_{\ell m}^{\prime}(t)\right|^{\rho} u_{\ell m}^{\prime}(t), w\right)=0, \text { for all } w \in W_{m}^{\ell}
\end{aligned}
$$


with the initial conditions

$$
u_{\ell m}(0)=u_{\ell}^{0}, \quad u_{\ell m}^{\prime}(0)=u_{\ell}^{1} .
$$

Taking into account (3.2) and denoting $M\left(\left|u_{l m}(t)\right|^{2}\right)$ by $\mu(t)$, we rewrite (3.8) as $\frac{\left(u_{\ell m}^{\prime \prime}(t), w\right)}{\mu(t)}+\left(\left(u_{\ell m}(t), w\right)\right)+\int_{\Gamma 1} \delta u_{\ell m}^{\prime}(t) w d \Gamma+\frac{\left(\left|u_{\ell m}^{\prime}(t)\right|^{\rho} u_{\ell m}^{\prime}(t), w\right)}{\mu(t)}=0$.

Notice that the solution $u_{\ell m}$ defined on $\left[0, t_{m}\right.$ [can be extended to the interval $[0, T]$, for any real number $T>0$, by the next first a priori estimate. We need two a priori estimates. First a Priori Estimate- By choosing $w=2 u_{\ell m}^{\prime}(t)$ in (3.10) we obtain

$$
\begin{aligned}
& \frac{d}{d t}\left[\frac{\left|u_{\ell m}^{\prime}(t)\right|^{2}}{\mu(t)}+\left\|u_{\ell m}(t)\right\|^{2}\right]+2 \int_{\Gamma 1} \delta\left(u_{\ell m}^{\prime}(t)\right)^{2} d \Gamma+ \\
& \frac{2}{\mu(t)}\left(\left|u_{\ell m}^{\prime}(t)\right|^{\rho} u_{\ell m}^{\prime}(t), u_{\ell m}^{\prime}(t)\right)=-\frac{\mu^{\prime}(t)}{\mu(t)^{2}}\left|u_{\ell m}^{\prime}(t)\right|^{2},
\end{aligned}
$$

whence by using (3.1) and (3.2) it follows that

$$
\begin{gathered}
\frac{d}{d t}\left[\frac{\left|u_{\ell m}^{\prime}(t)\right|^{2}}{\mu(t)}+\left\|u_{\ell m}(t)\right\|^{2}\right]+2 \int_{\Gamma 1} \delta\left(u_{\ell m}^{\prime}(t)\right)^{2} d \Gamma+ \\
\frac{2}{\mu(t)}\left\|u_{\ell m}^{\prime}(t)\right\|_{L^{\rho+2}(\Omega)}^{\rho+2} \leq \frac{2 K_{0}}{\mu(t)}\left|u_{\ell m}^{\prime}(t)\right|^{3},
\end{gathered}
$$

Moreover, since $L^{\rho+2(\Omega)} \rightarrow L^{2}(\Omega)$, there exists $C_{1}=C_{1}\left(K_{0}, \Omega\right)$ such that

$$
\frac{2 K_{0}}{\mu(t)}\left|u_{\ell m}^{\prime}(t)\right|^{3} \leq \frac{2 C_{1}}{\mu(t)}\left\|u_{\ell m}^{\prime}(t)\right\| \|_{L^{\rho+2}(\Omega)}^{3} \cdot
$$

The use of the Young's inequality, for all $\varepsilon>0$, yields

$$
\begin{aligned}
2 C_{1}\left\|u_{\ell m}^{\prime}(t)\right\|_{L^{\rho+2}(\Omega)}^{3} \leq & \frac{\rho-1}{\rho+2} \frac{\left(2 C_{1}\right)^{\frac{\rho+2}{\rho-1}}}{\varepsilon^{\frac{3}{\rho+2}}}+\frac{3 \varepsilon}{\rho+2}\left\|u_{\ell m}^{\prime}(t)\right\| \|_{L^{\rho+2}(\Omega)}^{\rho+2} \\
& \leq C_{2}(\varepsilon)+C_{3} \varepsilon\left\|u_{\ell m}^{\prime}(t)\right\| \begin{array}{l}
\rho+2 \\
L^{\rho+2}(\Omega)
\end{array} .
\end{aligned}
$$


By choosing a suitable $\varepsilon>0$, we have $\frac{d}{d t}\left[\frac{\left|u_{\ell m}^{\prime}(t)\right|^{2}}{\mu(t)}+\left\|u_{\ell m}(t)\right\|^{2}\right]+2 \int_{\Gamma 1} \delta\left(u_{\ell m}^{\prime}(t)\right)^{2} d \Gamma+\frac{C_{4}}{\mu(t)}\left\|u_{\ell m}^{\prime}(t)\right\| \|_{L^{\rho+2}(\Omega)}^{\rho+2} \leq C_{2}(\varepsilon)$.

Integrating on $\left[0, t\left[\right.\right.$ with $0<t<t_{m}$, by the Gronwall inequality and convergences (3.5) (3.6), for all $0 \leq t \leq T$ and $\ell \geq \ell_{0}$, we obtain

$$
\begin{aligned}
& \frac{\left|u_{\ell m}^{\prime}(t)\right|^{2}}{\mu(t)}+\left\|u_{\ell m}(t)\right\|^{2}+2 \int_{0}^{t} \int_{\Gamma 1} \delta\left(u_{\ell m}^{\prime}(s)\right)^{2} d s d \Gamma+ \\
& C_{4} \int_{0}^{t} \frac{\left\|u_{\ell m}^{\prime}(s)\right\| \begin{array}{l}
\rho+2 \\
L^{\rho+2}(\Omega)
\end{array}}{\mu(s)} d s \leq C_{2}(\varepsilon) T+\frac{\left|u^{1}\right|^{2}}{\mu(0)}+\left\|u^{0}\right\|^{2} .
\end{aligned}
$$

Thus for $m \in N$ and $\ell \geq \ell_{0}$ it follows that

$$
\begin{aligned}
& \left(u_{\ell m}\right) \text { is bounded in } L_{l o c}^{\infty}(0, \infty ; V), \\
& \left(u_{\ell m}^{\prime}\right) \text { is bounded in } L_{l o c}^{\infty}\left(0, \infty ; L^{2}(\Omega)\right), \\
& \left(\delta^{1 / 2} u_{\ell m}^{\prime}\right) \text { is bounded in } L_{l o c}^{2}\left(0, \infty ; L^{2}\left(\Gamma_{1}\right)\right), \\
& \left(u_{\ell m}^{\prime}\right) \text { is bounded in } L_{l o c}^{\rho+2}\left(0, \infty ; L^{\rho+2}(\Omega)\right),
\end{aligned}
$$

Note that in the obtention of $(3.12)_{2}$ we have used the fact $M \in C^{1}\left(\left[0, \infty[)\right.\right.$ and $(3.12)_{1}$.

Second a Priori Estimate - In order to obtain estimate for $u_{\ell m}^{\prime \prime}(t)$, we differenciate (3.10) with respect to $\mathrm{t}$ and then we choose $w=2 u_{l m}^{\prime \prime}(t)$. So, we obtain

$$
\begin{aligned}
& \frac{d}{d t}\left[\frac{\left|u_{\ell m}^{\prime \prime}(t)\right|^{2}}{\mu(t)}+\left\|u_{\ell m}^{\prime}(t)\right\|^{2}\right]+2 \int_{\Gamma 1} \delta\left(u_{\ell m}^{\prime \prime}(t)\right)^{2} d \Gamma+\frac{\rho+1}{\mu(t)}\left(\left|u_{\ell m}^{\prime}(t)\right|^{\rho},\left(u_{\ell m}^{\prime \prime}(t)\right)^{2}\right)= \\
& \frac{2 \mu^{\prime}(t)}{\mu(t)^{2}}\left|u_{\ell m}^{\prime \prime}(t)\right|^{2}+\frac{2 \mu^{\prime}(t)}{\mu(t)^{2}}\left(\left|u_{\ell m}^{\prime}(t)\right| \rho u_{\ell m}^{\prime}(t), u_{\ell m}^{\prime \prime}(t)\right) \text {. }
\end{aligned}
$$


Note that for $\varepsilon>0$

$$
\begin{aligned}
& \left(\left|u_{\ell m}^{\prime}(t)\right|^{\rho} u_{\ell m}^{\prime \prime}(t), u_{\ell m}^{\prime \prime}(t)\right)=\left(\left|u_{\ell m}^{\prime}(t)\right|^{\frac{\rho}{2}} u_{\ell m}^{\prime \prime}(t), \mid u_{\ell m}^{\prime}(t)^{\frac{\rho}{2}} u_{\ell m}^{\prime}(t)\right) \\
& \leq \frac{\varepsilon}{2}\left(\left|u_{l m}^{\prime}(t)\right|^{\rho},\left(u_{\ell m}^{\prime \prime}(t)^{2}\right)\right)+\frac{1}{2 \varepsilon}\left\|u_{l m}^{\prime}(t)\right\|_{L^{\rho+2}(\Omega)}^{\rho+2} .
\end{aligned}
$$

So,

$$
\begin{aligned}
& \frac{d}{d t}\left[\frac{\left|u_{\ell m}^{\prime \prime}(t)\right|^{2}}{\mu(t)}+\left\|u_{l m}^{\prime}(t)\right\|^{2}\right]+2 \int_{\Gamma 1} \delta\left(u_{l m}^{\prime \prime}(t)\right)^{2} d \Gamma+ \\
& \left(\frac{\rho+1}{\mu(t)}-\varepsilon C\right)\left(\left|u_{\ell m}^{\prime}(t)\right|^{\rho},\left(u_{\ell m}^{\prime \prime}(t)\right)^{2}\right) \leq 2 C \frac{\left|u_{l m}^{\prime \prime}(t)\right|^{2}}{\mu(t)}+\frac{1}{2 \varepsilon}\left\|u_{l m}^{\prime}(t)\right\| \|_{L^{\rho+2}(\Omega)}^{\rho+2} .
\end{aligned}
$$

Taking a suitable $\varepsilon$ and integrating on $\left[0, t\left[\right.\right.$, for all $\ell \geq \ell_{0}$, we get

$$
\begin{aligned}
& \frac{\left|u_{\ell m}^{\prime \prime}(t)\right|^{2}}{\mu(t)}+\left\|u_{\ell m}^{\prime}(t)\right\|^{2}+2 \int_{0}^{t} \int_{\Gamma 1} \delta\left(u_{\ell m}^{\prime \prime}(s)\right)^{2} d \Gamma d s+ \\
& C_{0} \int_{0}^{t}\left(\left|u_{\ell m}^{\prime}(s)\right|^{\rho},\left(u_{\ell m}^{\prime \prime}(s)\right)^{2}\right) \leq \frac{\left|u_{\ell m}^{\prime \prime}(0)\right|^{2}}{\mu(0)}+ \\
& \left\|u^{1}\right\|^{2}+2 C \int_{0}^{t} \frac{\left|u_{\ell m}^{\prime \prime}(s)\right|^{2}}{\mu(s)} d s+\frac{1}{2 \varepsilon} \int_{0}^{t}\left\|u_{\ell m}^{\prime}(s)\right\| \|_{L^{\rho+2}(\Omega)}^{\rho+2} d s .
\end{aligned}
$$

To finish the second estimate we need to bound $\left(u_{l m}^{\prime \prime}(0)\right)$ in $L^{2}(\Omega)$. In this point becomes clear the importance of the special basis that we have constructed. In fact, we make $t=0$ in (3.10) and take $w=u_{\ell m}^{\prime \prime}(0)$. This yields

$$
\frac{\left|u_{\ell m}^{\prime \prime}(0)\right|^{2}}{\mu(0)}+\left(\left(u_{\ell m}(0), u_{\ell m}^{\prime \prime}(0)\right)\right)+\int_{\Gamma 1} \delta u_{\ell m}^{1} u_{\ell m}^{\prime \prime}(0) d \Gamma+\frac{\left(\left|u_{\ell}^{1}\right|^{\rho} u_{\ell}^{1}, u_{\ell m}^{\prime \prime}(0)\right)}{\mu(0)}=0 .
$$

By using Green's Theorem we obtain

$$
\frac{\left|u_{\ell m}^{\prime \prime}(0)\right|^{2}}{\mu(0)}=\left(\Delta u_{\ell}^{0}, u_{\ell m}^{\prime \prime}(0)\right)-\int_{\Gamma 1}\left(\frac{\partial u_{\ell}^{0}}{\partial v}+\delta u_{\ell}^{1}\right) u_{\ell m}^{\prime \prime}(0) d \Gamma-\frac{\left(\left|u_{\ell}^{1}\right|^{\rho} u_{\ell}^{1}, u_{\ell m}^{\prime \prime}(0)\right)}{\mu(0)}
$$




$$
\frac{\left|u_{\ell m}^{\prime \prime}(0)\right|^{2}}{\mu(0)}=\left(\Delta u_{\ell}^{0}, u_{\ell m}^{\prime \prime}(0)\right)-\int_{\Gamma 1}\left(\frac{\partial u_{\ell}^{0}}{\partial v}+\delta u_{\ell}^{1}\right) u_{\ell m}^{\prime \prime}(0) d \Gamma-\frac{\left(\left|u_{\ell}^{1}\right|^{\rho} u_{\ell}^{1}, u_{\ell m}^{\prime \prime}(0)\right)}{\mu(0)}
$$

and by using that $\frac{\partial u_{\ell}^{0}}{\partial v}+\delta u_{\ell}^{1}=0$ on $\Gamma_{1}$, we get

$$
\left|u_{\ell m}^{\prime \prime}(0)\right| \leq \mu(0)\left[\left|\Delta u_{\ell}^{0}\right|+\left\|u_{\ell}^{1}\right\|_{L^{2(\rho+1)}(\Omega)}^{\rho+1}\right] .
$$

Then, taking into account (3.3) and (3.4), we have $V \hookrightarrow L^{2(\rho+1)}(\Omega)$ and therefore

$$
\left|u_{\ell m}^{\prime \prime}(0)\right| \leq C \mu(0)\left[\left|\Delta u_{\ell}^{0}\right|+\left\|u_{\ell}^{1}\right\|^{\rho+1}\right] .
$$

Combining the above inequality with (3.14) and (3.12), for $m \in N$ and $\ell \geq \ell_{0}$, we get:

$$
\begin{aligned}
& \left(u_{\ell m}^{\prime}\right) \text { is bounded in } L_{l o c}^{\infty}(0, \infty ; V), \\
& \left(u_{\ell m}^{\prime \prime}\right) \text { is bounded in } L_{l o c}^{\infty}\left(0, \infty ; L^{2}(\Omega)\right), \\
& \left(\delta^{1 / 2} u_{\ell m}^{\prime \prime}\right) \text { is bounded in } L_{l o c}^{2}\left(0, \infty ; L^{2}\left(\Gamma_{1}\right)\right)
\end{aligned}
$$

Estimates (3.12) and (3.15) allows us, by induction and diagonal process, to obtain a subsequence $\left(u_{\ell m}^{(p)}\right)$ of $\left(u_{\ell m}\right)$ which will be also denoted by $\left(u_{\ell m}\right)$, and a function $u: \Omega \times] 0, \infty[\rightarrow R$ satisfying:

$$
\begin{aligned}
& u_{\ell m} \rightarrow u \text { weak star in } L_{l o c}^{\infty}(0, \infty ; V), \\
& u_{\ell m}^{\prime} \rightarrow u^{\prime} \text { weak star in } L_{l o c}^{\infty}(0, \infty ; V), \\
& u_{\ell m}^{\prime} \rightarrow u^{\prime} \text { weak star in } L_{l o c}^{\rho+2}\left(0, \infty ; L^{\rho+2}(\Omega)\right), \\
& u_{\ell m}^{\prime \prime} \rightarrow u^{\prime \prime} \text { weak star in } L_{l o c}^{\infty}\left(0, \infty ; L^{2}(\Omega)\right), \\
& \delta^{1 / 2} u_{\ell m}^{\prime \prime} \rightarrow X \text { weakly in } L_{l o c}^{2}\left(0, \infty ; L^{2}\left(\Gamma_{1}\right)\right)
\end{aligned}
$$

and as a consequence 


$$
\delta^{1 / 2} u_{\ell m}^{\prime} \rightarrow \delta^{1 / 2} u^{\prime} \text { weak star in } L_{l o c}^{\infty}\left(0, \infty ; H^{1 / 2}\left(\Gamma_{1}\right)\right) .
$$

Convergences (3.16) and (3.17) allow us to pass to the limit in (3.7). Moreover, by using the regularity (3.16) of $u$, we obtain

$$
u^{\prime \prime}-M\left(|u|^{2}\right) \Delta u+\left|u^{\prime}\right|^{\rho} u^{\prime}=0 \quad \text { in } \quad L_{l o c}^{\infty}\left(0, \infty ; L^{2}(\Omega)\right)
$$

From the assumptions (3.3) and (3.4), it follows that $V G L^{2(\rho+1)}(\Omega)$. . So, we take into account (3.18) to deduce that $\Delta u \in L_{l o c}^{\infty}\left(0, \infty ; L^{2}(\Omega)\right.$; and as $u \in L^{\infty}(0, \infty ; V)$, we get

$$
\frac{\partial u}{\partial v} \in L_{l o c}^{\infty}\left(0, \infty ; H^{-1 / 2}(\Gamma)\right) .
$$

Since $W$ is dense in $V$, after to pass to the limit in (3.8), we obtain

$$
\begin{aligned}
& \int_{0}^{\infty}\left(u^{\prime \prime}, v\right) \theta d t+\int_{0}^{\infty} M\left(|u(.)|^{2}\right)((u, v)) \theta d t+ \\
& \int_{0}^{\infty} M\left(|u(.)|^{2}\right) \int_{\Gamma_{1}} \delta u^{\prime} v \theta d \Gamma d t+\int_{0}^{\infty}\left(\left|u^{\prime}\right|^{\rho} u^{\prime}, v\right) \theta d t=0,
\end{aligned}
$$

for all $v \in V$ and for all $\theta \in D(0, \infty)$. On the other hand, multiplying (3.18) by $v \theta$ with $v \in V$ and $\theta \in D(0, \infty)$ integrating and using Green's Theorem, we have

$$
\begin{aligned}
& \int_{0}^{\infty}\left(u^{\prime \prime}, v\right) \theta d t+\int_{0}^{\infty} M\left(|u(.)|^{2}\right)((u, v)) \theta d t- \\
& -\int_{0}^{\infty} M\left(\mid u\left(.\left.\right|^{2}\right)\left\langle\frac{\partial u}{\partial v}, v\right\rangle \theta d t+\int_{0}^{\infty}\left(\left|u^{\prime}\right|{ }^{\rho} u^{\prime}, v\right) \theta d t=0,\right.
\end{aligned}
$$

where $\langle.,$.$\rangle denotes the duality pairing between of H^{-1 / 2}(\Gamma)$ and $H^{1 / 2}(\Gamma)$. So, comparing (3.19) with (3.20) we have

$$
\int_{0}^{\infty}\left\langle M\left(|u(.)|^{2}\right)\left[\frac{\partial u}{\partial v}+\delta v^{\prime}\right], \psi\right\rangle_{H^{-1 / 2}(\Gamma), H^{1 / 2}(\Gamma)} \theta d t=0,
$$

for all $\psi \in D\left(\Gamma_{1}\right)$ and $\theta \in D([0, \infty[)$. This and regularity (3.17) imply 


$$
\frac{\partial u}{\partial v}+\delta v^{\prime}=0 \quad \text { in } \quad L_{l o c}^{\infty}\left(0, \infty ; H^{1 / 2}\left(\Gamma_{1}\right)\right)
$$

From above equality we can conclude that $g_{u} \equiv \delta u^{\prime}$. Therefore $u \in L_{l o c}^{\infty}(0, \infty ; W)$. Moreover, as shown in [4], from (3.21) it follows that

$$
\frac{\partial u^{\prime}}{\partial v}+\delta u^{\prime \prime}=0 \quad \text { in } \quad H_{l o c}^{-1}\left(0, \infty ; H^{-1 / 2}\left(\Gamma_{1}\right)\right)
$$

but by $(3.16)_{6}$ we have $\delta u^{\prime \prime} \in L_{l o c}^{2}\left(0, \infty ; L^{2}\left(\Gamma_{1}\right)\right)$, therefore the last equality is verified in the space $L_{l o c}^{2}\left(0, \infty ; L^{2}\left(\Gamma_{1}\right)\right)$.

Uniqueness of solutions and the verification on the initial conditions are showed by the standard arguments.

\section{REFERENCES}

1. Carrier, G. F., On the non-linear vibration problem of the elastic string, Quart. Appl. Math. 3(1945), pp. 157-165.

2. Cousin, A. T., Frota, C. L., Lar'kin, N. A. and Medeiros, L. A., On the abstract model of the Kirchhoff-Carrier equation, Comm. Appl. Analysis, 1(1997), pp. 389-404.

3. Frota, C. L., Cousin, A. T. and Lar'kin, N. A., Existence of global solutions and energy decay for the Carrier equation with dissipative term, Notas do $45^{\circ}$ SBA, (1997), pp. 247-251.

4. Milla Miranda, M., Traço para o Dual dos Espaços de Sobolev, Bol. Soc. Paran. Matemática (2º́rie), 11, 2(1990), pp. 131-157.

5. Milla Miranda, M. and Medeiros, L. A., On a Boundary Value Problem for Wave Equations: Existence, Uniqueness-Asymptotic Behavior, Revista de Matemáticas Aplicadas, Universidad de Chile, 17(1996), pp. 47-73.

6. Milla Miranda, M. and San Gil Jutuca, L. P., On global existence of solution for a damped non-linear wave equation, Notas do $46^{\circ} \mathrm{SBA}$, (1997), pp. 619-624. 